\title{
Investigation of Full-field and Mean-field Models for Pure Grain Growth Simulations
}

\author{
Betul Aktas ${ }^{1}$ (D) Caner Simsir ${ }^{2}$ (D) \\ ${ }^{1}$ Tampere University, Department of Materials Science Engineering, Tampere, Finland \\ ${ }^{2}$ Middle East Technical University, Department of Metallurgical and Materials Engineering, Ankara, Turkey
}

\section{A B S T R ACT}

$\mathrm{M}$ ean-field approaches are commonly used in the simulation of grain growth in metals as they are easier to implement. However, mean-field models only track the evolution of average grain diameter as a function of temperature and time, while they neglect the effect of actual grain size distribution, which limits their applicability. Recently introduced full-field models of grain growth - either by level-set and phase field methods- allow to overcome the barriers of mean-field methods, but they are computationally much more demanding. In this article, the main goal is to investigate the applicability of both approaches for the pure grain growth in a solid with an initial Gaussian grain size distribution. The main idea is to keep the average grain size constant while altering the grain size distribution by modifying the standard deviation. DIGIMU software, which uses the level-set approach - is used for this purpose. The conclusion is that full-field models are beneficial to observe changes during grain growth; alternatively, mean-field models deduce approximately the same results as full-field models for a Gaussian distribution within a shorter time. However, it is found that mean-field models overlook certain important stages of the evolution of the microstructure, while full-field method captures all the details. Therefore, the model to investigate the grain growth mechanism should be selected accordingly.

Keywords: Grain growth, Mean-field models, Full-field models, Mean grain size

\section{Article History:}

Received: 2020/11/15

Accepted: 2021/03/15

Online: 2021/03/31

Correspondence to: Caner Şimşir

E-mail:csimsir@metu.edu.tr;

Phone: +9o (312) 210-2520;

Fax: +90 (312) 210-5918.

\section{INTRODUCTION}

G rain growth is a phenomenon during which the 7 average grain size gets larger to decrease the total area of grain boundaries to decrease the total energy of the material. Before the grain growth, other mechanisms such as recovery or recrystallization take place to lower the energy; however, further energy decrease is achieved by grain growth at higher temperatures [1]. Change in the grain diameter has a significant effect on the processing and the design of the material due to the fact that material property is affected by grain size [2]. Experts from different backgrounds have studied the change in the material properties like mechanical [3], dielectric [4], and magnetic [5] as a function of grain size over the years. Therefore, much research is going on about the simulation of the growth processes. The easiest growth phenomenon is the pure or ideal grain growth, which does not include other factors such as recrystallization and grain boundary (Smith-Zener) pinning effect [6].
The kinetics of boundary migration must be well understood to study grain growth. Grain boundary migration occurs due to the curved surface of the grains under the existence of an internal pressure. In principle, the migration is towards the center of curvature of grain boundaries. This phenomenon enables the reduction in the overall inner interface and consequently minimizes the overall boundary energy [7]. The kinetics of normal grain growth is generally represented by Eq. (1)

$$
\langle R\rangle^{2}-\langle R\rangle_{0}^{2}=k . t
$$

where $\mathrm{t}$ is the time, $\langle R\rangle$ is the average grain radius at $\mathrm{t},\langle R\rangle_{0}$ is the initial value of $\langle R\rangle$ at $\mathrm{t}=0$, and $\mathrm{k}$ is the kinetic coefficient.

Mathematical models of grain growth allow us to perform simulations. There are many types of grain growth models; however, full-field and mean-field models are the most popular ones. Each model has some drawbacks and conditions that make it useful 
for different applications. Mean-field models work with the average values of grain size and overlook the grain size distribution. It means that providing only the average grain sizes to the software is enough to obtain the final average grain sizes. However, full-field models focus on the grain size distribution; therefore, it requires a whole picture of the domain. In terms of reality, full-field methods include more information about the material than the mean-field methods. On the other hand, this high level of detailed information is accompanied by very high computational costs [8]. Calibration of numerical parameters, whose number is also increasing in a 3D study, is another difficult problem to deal with. Due to all these reasons, especially in the computation of 3D models, fullfield models have issues waiting to be solved. Some recent progress addresses those issues to prevent obstacles in their industrial use [9] [10]. Furstoss et al. [11] provide a full-field method to simulate grain growth in multiphase materials. Their model can construct transient and steady-state structures based on the level-set approach. After using past experimental data to limit their full-field model, their analyses showed that experimental microstructure morphologies differ from simulated morphologies. Using mean-field and full-field models together, they could predict the mean grain size growth from an experimental peridotite structure.

\section{MATERIAL AND METHODS}

DIGIMU $^{\circledR}$ is a software that enables the user to simulate different kinds of phenomena happening in the microstructure. In grain growth simulations, DIGIMU ${ }^{\bullet}$ uses Level Set functions inside the finite element computations. Grain boundaries, interfaces, are defined with the Level Set functions so that each function represents a grain boundary. Level Set function, $\psi$, can be named as a signed distance function, which is defined in Eq. (2) over a domain $\Omega$. $\Omega$ has many sub-domains denoted by G. Distance is calculated as the Euclidean distance, $\Delta$, to the interface, $\Gamma$, of sub-domains. In finite element mesh, $\psi$ values are calculated for each node points, and depending on whether $\psi>0$ or $\psi<0$, the point is said to lay inside or outside, respectively. Interfaces are also defined by the level set functions in Eq. (3), where the distance to itself is defined as zero.

$$
\begin{aligned}
& \psi(x, t)= \pm \Delta(x, \Gamma) \\
& \Gamma(t)=\psi(x, t)=0
\end{aligned}
$$

In terms of computational expenses, defining each grain boundary with a level set function is costly. In order to reduce the numerical cost, a coloring technique is used. In this way, grain boundaries that are represented with the same level set function define grains with same colors, only if they are not adjacent. In the end, computational costs are minimized due to the decrease in the total number of level set functions.

In order to determine the grain boundary velocity, $\vec{v}_{c}$, grain boundary mobility, $M_{b}$, should be calculated. Based on simulation time increments, the derivative of distance function, denoted by $\Delta \psi$, is also used to calculate grain boundary velocity. The equations required to calculate those parameters are given in Eq. (4) and Eq. (5).

$$
\begin{aligned}
& \vec{v}_{c}=-M_{b} \gamma_{b} \Delta \psi \vec{\nabla} \psi \\
& M_{b}(T)=M_{0} \exp \left(\frac{-Q_{m}}{R T}\right)
\end{aligned}
$$

where $Q_{m}$ is the constant activation energy, $M_{0}$ is the mobility pre-exponential factor; and $\gamma_{b}$ is the grain boundary energy. Those parameters are material specific and can be found in the material file for Inconel 718, also tabulated in Table 1.

Table 1. Material data of INCONEL 718 for full-field grain growth simulation in DIGIMU. $\mathrm{M}_{0}$ : mobility pre-exponential factor, $\mathrm{Q}_{\mathrm{m}}$ : constant activation energy, $\gamma_{\mathrm{b}}$ : grain boundary energy (Data is taken from the DIGIMU 3.0 material library.)

\begin{tabular}{ccc}
\hline $\begin{array}{c}M_{o} \\
\left(\mathrm{~mm}^{4} / \mathrm{Js}\right)\end{array}$ & $\begin{array}{c}Q_{m} \\
(\mathrm{~J} / \mathrm{mol})\end{array}$ & $\begin{array}{c}\gamma_{b} \\
\left(\mathrm{~J} / \mathrm{mm}^{2}\right)\end{array}$ \\
\hline $2.981 \times 10^{15}$ & 387000 & $6 \times 10^{-7}$ \\
\hline
\end{tabular}

Mean-field and full-field approaches are investigated by performing two simulations using DIGIMU 3.0. The main idea is to generate two initial polycrystals having different grain size distributions and the same mean grain size so that it is possible to observe differences between the approaches. Simulations are performed in three stages, pre-processing, computation, and post-processing.

It is known that $\delta$ phases precipitate at temperatures lower than $1050-1100{ }^{\circ} \mathrm{C}[12]$ and precipitates activate other mechanisms like Zener pinning during the grain growth. Therefore, $1100{ }^{\circ} \mathrm{C}$ is suitable as a heat treatment temperature to avoid precipitation since the aim is to demonstrate

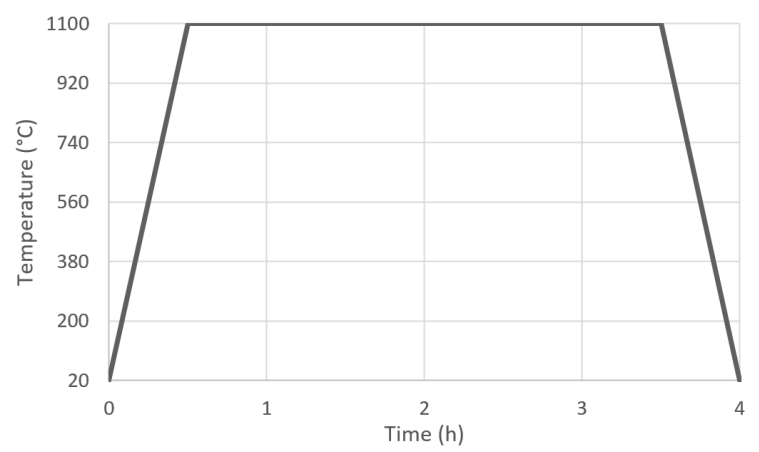

Figure 1. The thermal cycle of the simulations. 
pure grain growth. Parameters are defined so that the process lasts 4 hours, a heating step of $1 / 2$ hour between $20^{\circ} \mathrm{C}$ and $1100{ }^{\circ} \mathrm{C}$ followed by a 3 hours step at this same temperature and then a $1 / 2$ our cooling step between $1100{ }^{\circ} \mathrm{C}$ and $20^{\circ} \mathrm{C}$. The thermal cycle of the process can be seen in Fig. 1.

Representative elementary volume (RVE) is defined as a square with dimensions $3 \mathrm{~mm} \times 3 \mathrm{~mm}$ in 2D. The software automatically generated 1139 sites (grains) in RVE. Standard deviations are determined as $5 \mu \mathrm{m}$ and $15 \mu \mathrm{m}$ to obtain dissimilar grain size distributions. The mean grain size is decided to be set as $50 \mu \mathrm{m}$ because it is the typical value for this alloy, which is specified in AMS 5663[13]. The minimum and maximum grain sizes allowed in the initial polycrystals are given together with the above criteria in Table 2.

Table 2. The statistical data to form grain size distributions for each simulation. (GS: Grain Size)

\begin{tabular}{lcccc}
\hline & Min.G.S. & Max.G.S. & MeanG.S & Standard Deviation \\
\hline Simulation 1 & $5 \mu \mathrm{m}$ & $90 \mu \mathrm{m}$ & $50 \mu \mathrm{m}$ & $5 \mu \mathrm{m}$ \\
Simulation 2 & $5 \mu \mathrm{m}$ & $90 \mu \mathrm{m}$ & $50 \mu \mathrm{m}$ & $15 \mu \mathrm{m}$ \\
\hline
\end{tabular}

After creating the project file and defining the simulation parameters, the project is launched to compute. Each of the simulations lasted approximately 150 minutes. Results are obtained as microstructural figures and as grain size distribution histograms.

\section{RESULTS AND DISCUSSION}

To investigate the grain boundary movement and the grain size alteration, frames at selected times are given in Fig. 2 and Fig. 3, which show distances to grain boundaries from the center of the grains for each simulation. Since distributions are different, starting microstructures are also different from each other. The grains in Simulation 1 when $\mathrm{t}=0 \mathrm{~s}$ are smaller than in Simulation 2, which was expected before the simulation due to differences in the distributions.

By analyzing the reddish spots, it is easier to detect larger grains in Fig. 2 and Fig. 3. It is possible to visualize any desired grain size by changing the color threshold. It should be noted that the colorization of the grains is performed separately in Simulation 1 and 2. Therefore, the maximum values marked by red color are not the same. The distributions in final microstructures need to be investigated since it is not easy to say how they are different from each other, unlike in the initial microstructure. Therefore, histograms of the grain size distributions are required to be analyzed deeply.

In Fig. 4, progress in Simulation 1 is shown by grain size distributions. It is possible to track the behavior of the distributions during the simulation by making use of the- se histograms. Since we defined the initial distribution as a normal distribution, the initial histogram $(t=0 \mathrm{~s})$ is in a shape similar to a regular bell curve. However, as time passes, grain size distributions deviate from that shape because grains with different sizes are formed more likely than the initial condition.

It is not very easy to follow mean grain size from these histograms; therefore, mean grain sizes are provided on each figure, and it is concluded that mean grain size increases during the simulation as the grain sizes are increasing. Moreover, not only that the mean grain size increases, but also the grain size range enlarges. For instance, the histogram at $\mathrm{t}=0 \mathrm{~s}$ shows that grain sizes are ranged from $37 \mu \mathrm{m}$ to $68 \mu \mathrm{m}$; while the histogram at $\mathrm{t}=14400 \mathrm{~s}$ shows the range is from $29 \mu \mathrm{m}$ to $279 \mu \mathrm{m}$. Therefore, the probability of observing larger grains increases as time passes.

In Simulation 2, a similar change in the histograms is seen in Fig. 5. It starts with a normal distribution, deviates from bell-shaped as time passes. The range of the grain sizes again increases during the simulation. In the beginning, grain sizes are ranged from $16 \mu \mathrm{m}$ to $83 \mu \mathrm{m}$. In the end, this range enlarges to $19 \mu \mathrm{m}-292 \mu \mathrm{m}$. Therefore, larger grains are likely to be found in the structure as time passes.

Initial distribution can be generated with higher nonuniformity in terms of grain size by keeping the mean grain size constant and increasing the standard deviation of the initial polycrystal grain size distribution, as concluded in Fig. 4 and Fig. 5. Even from the first step at $t=0$ s, grain sizes are allowed to exist in a broader range in Fig. 5 than that in Fig. 4 (from $37 \mu \mathrm{m}-68 \mu \mathrm{m}$ to $16 \mu \mathrm{m}-83 \mu \mathrm{m}$ ). Likewise, following the same trend, one can say that at $t=14400 \mathrm{~s}$, the grain size allowance range would be higher in Fig. 5 than that in Fig.4. It can be confirmed with the increase in the range from 19 $\mu \mathrm{m}-292 \mu \mathrm{m}$ to $29 \mu \mathrm{m}-279 \mu \mathrm{m}$.

In order to track mean grain size during the simulation, all the frames were analyzed and Fig. 6 is obtained. The increase in mean grain size during the simulations was expected since grains are getting larger. The heat treatment cycle can also be seen on the same figure to track the relation between the temperature and growth. The increase in the temperature affects grain growth since the grain boundary mobility is affected by temperature. Up to around $1000{ }^{\circ} \mathrm{C}$, average grain size is not changing because the temperature is not enough to activate grain growth. After that point on, grain growth accelerates and the average grain size visibly increases. At $t=12600 \mathrm{~s}$, when the cooling starts, growth is completed because the grain boundary mobility is too low due to the absence of heat. The mean grain sizes at the end of each simulation are given in Table 3 .

The main goal of these simulations is to determine 
the differences between mean-field and full-field models by carrying out two full-field model based simulations. It can be easily seen in Fig. 6 that both simulations reached approximately the same mean grain size at the end (Table 3). The slight difference is calculated as $1.7 \mu \mathrm{m}$. Therefore, mean and full-field models reach similar results by means of average grain size. However, the paths that simulations followed are a bit more different from each other. The maximum difference in the mean grain sizes between the simulations reached at $\mathrm{t}=4400 \mathrm{~s}$, and the difference is $13.25 \mu \mathrm{m}$, which is a non-negligible difference. This difference can be calculated since the full-field approach provides the advantage of tracking mean grain size evolution throughout the simulation, unlike the mean-field approach. Even though mean field approach provides promising results for the final

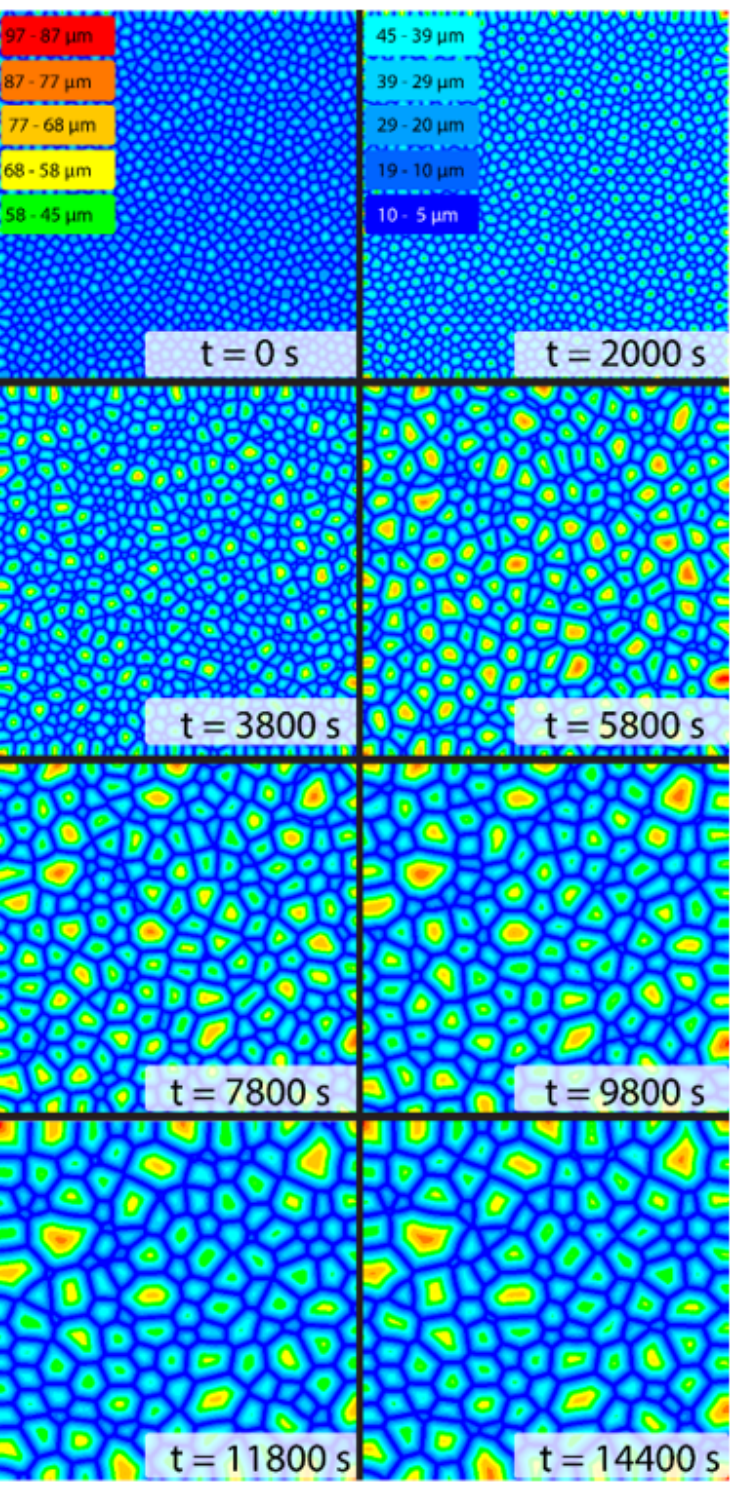

Figure 2. Distances to grain boundaries from the centers of the grains in Simulation 1. mean grain size, it cannot predict the grain size distribution and the evolution of mean grain size during the simulation. Therefore, a full-field approach should be used if the investigation aims to examine the structure of the material while the grain growth is operating. In other words, mean-field approaches can only provide final mean grain sizes, but it is not useful to determine the grain size distribution.

Table 3. Final mean grain size for each simulation and differences between mean grain sizes

\begin{tabular}{lcc}
\hline & Simulation 1 & Simulation 2 \\
\hline $\begin{array}{l}\text { Final mean grain size } \\
\begin{array}{c}\text { Final difference in the } \\
\text { mean grain sizes }\end{array}\end{array}$ & $130.4 \mu \mathrm{m}$ & $132.1 \mu \mathrm{m}$ \\
$\begin{array}{c}\text { Maximum difference in } \\
\text { the mean grain sizes }\end{array}$ & $1.7 \mu \mathrm{m}$ & \\
\hline
\end{tabular}

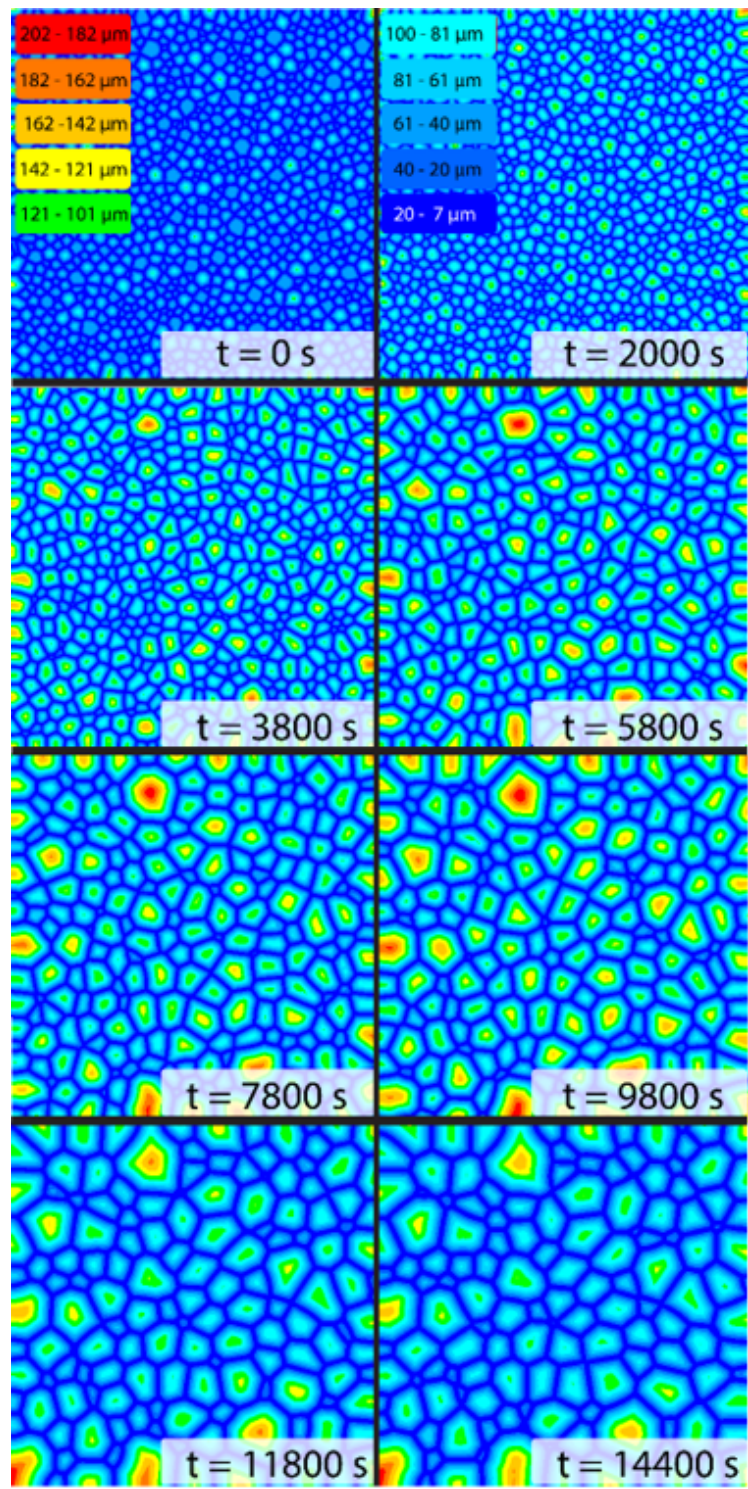

Figure 3. Distances to grain boundaries from the centers of the grains in Simulation 2 


$$
\mathrm{t}=\mathrm{Os}
$$

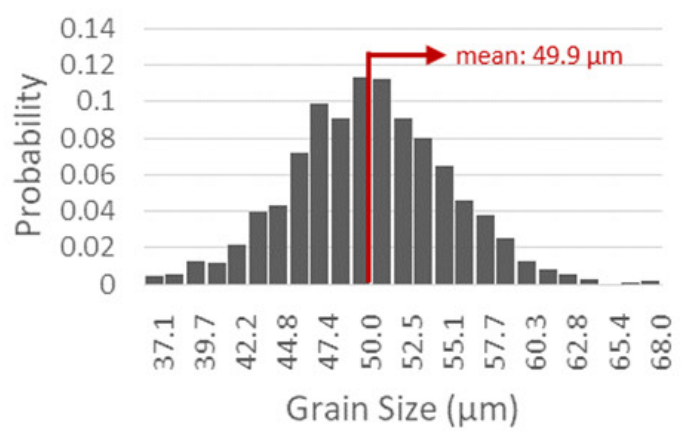

$$
t=3800 s
$$

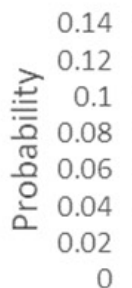

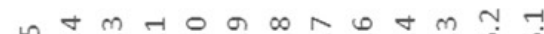

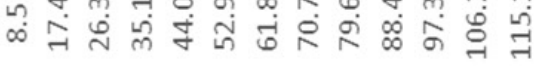
Grain Size $(\mu \mathrm{m})$

$$
t=7800 \mathrm{~s}
$$

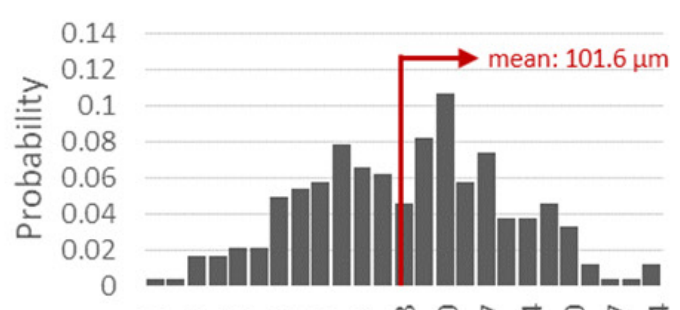

ก

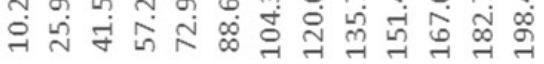
Grain Size $(\mu \mathrm{m})$

$$
\mathrm{t}=11800 \mathrm{~s}
$$

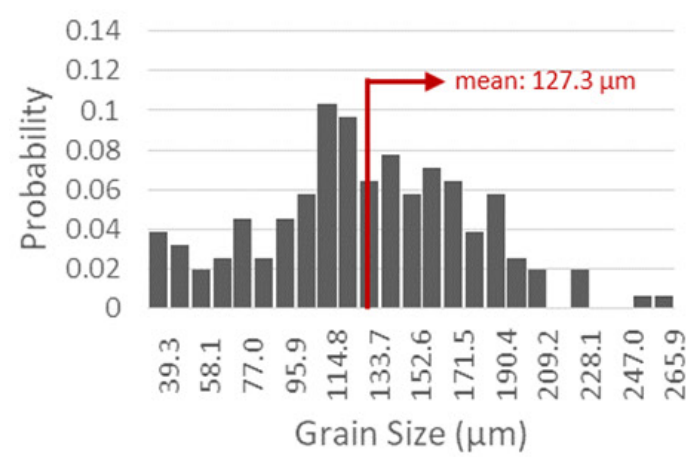

Figure 4. Grain size distribution throughout the Simulation 1. $\mathrm{t}=2000 \mathrm{~s}$

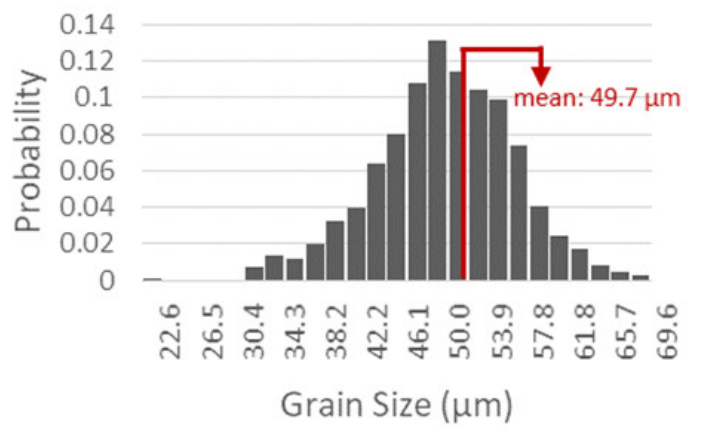

$t=5800 s$

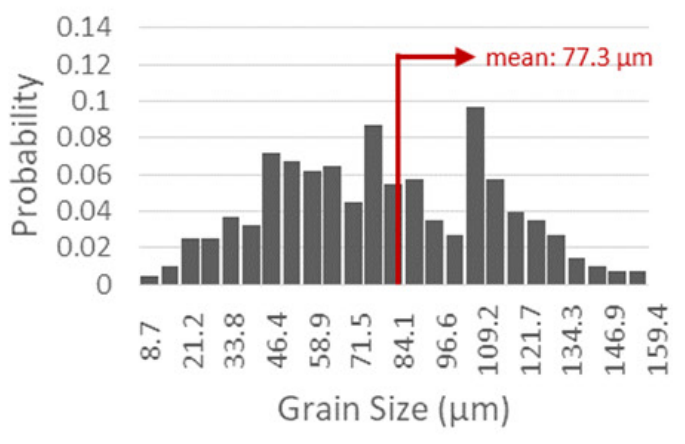

$t=9800 \mathrm{~s}$
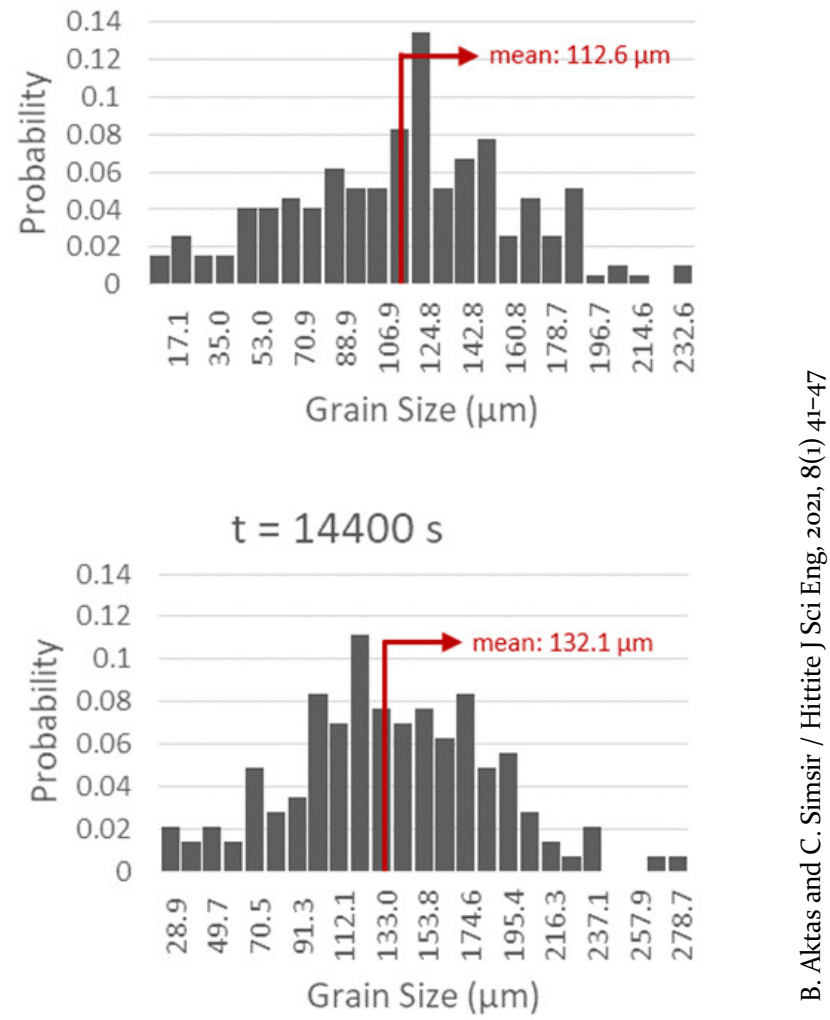


$$
\mathrm{t}=0 \mathrm{~s}
$$

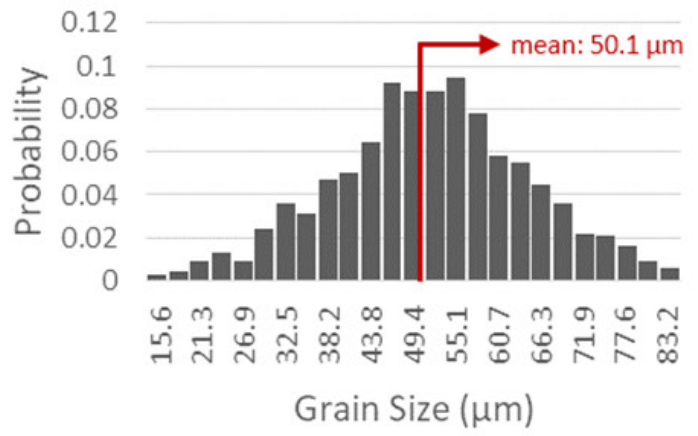

$\mathrm{t}=3800 \mathrm{~s}$

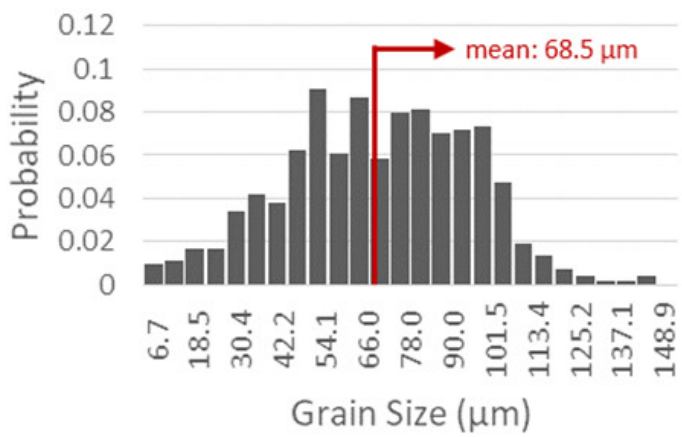

$$
t=7800 s
$$

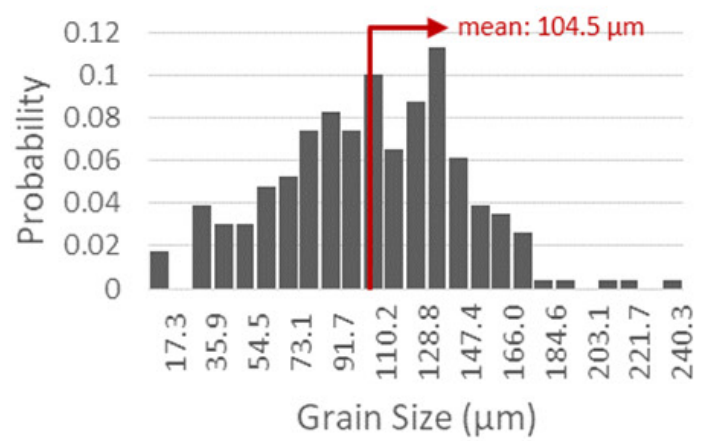

$t=11800 \mathrm{~s}$

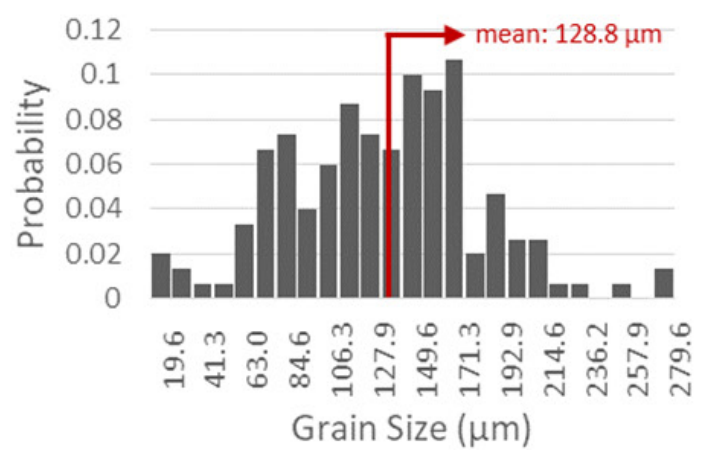

Figure 5. Grain size distribution throughout the Simulation 2.

\section{$t=2000 s$}

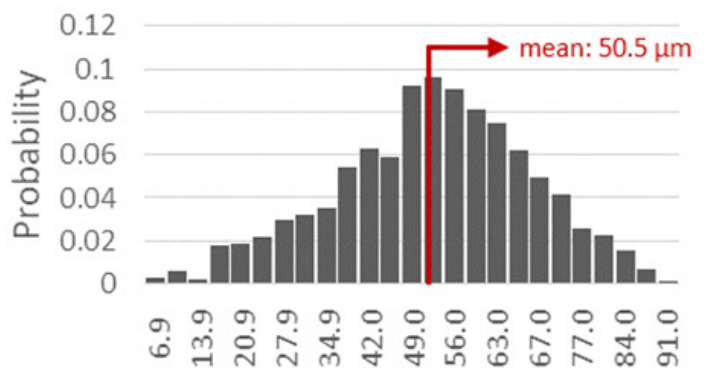

Grain Size $(\mu \mathrm{m})$

$$
t=5800 s
$$

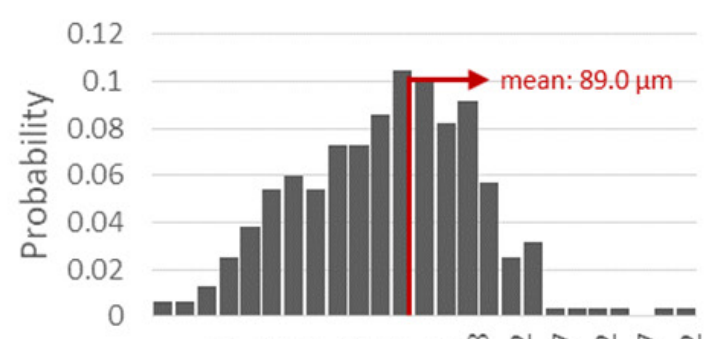

$m \infty m \infty m \infty n, \cdots$

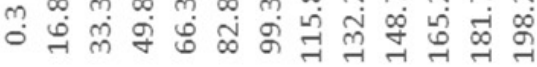
Grain Size $(\mu \mathrm{m})$

$$
\mathrm{t}=9800 \mathrm{~s}
$$

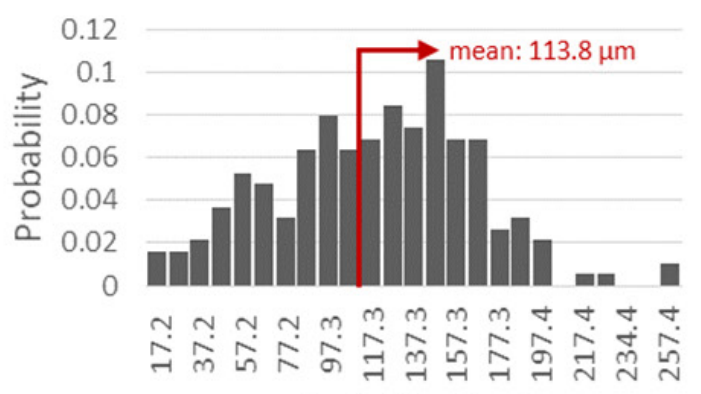
Grain Size ( $\mu \mathrm{m})$

$$
\mathrm{t}=14400 \mathrm{~s}
$$

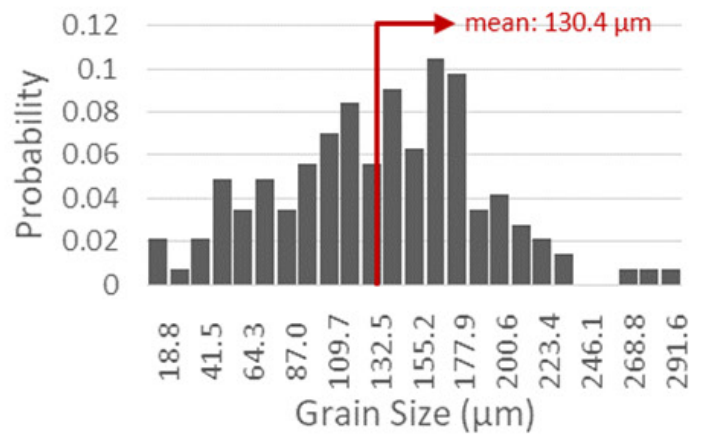




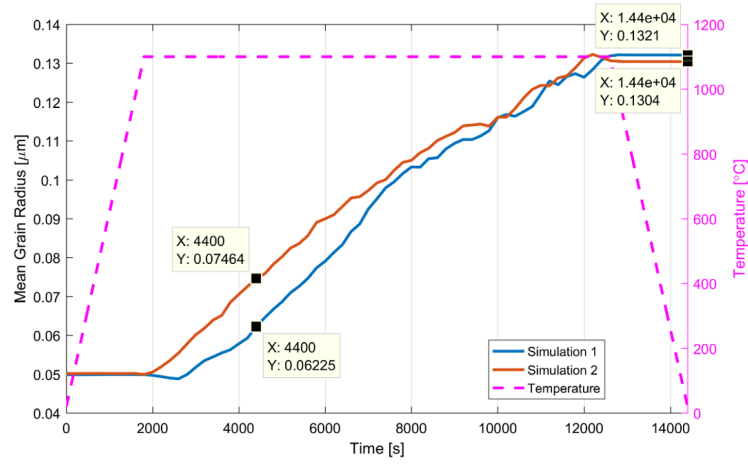

Figure 6. The change in the mean grain size during the simulations.

\section{CONCLUSION}

Investigation of the grain growth mechanism has been studied over the years due to the fact that change in the grain size has a huge impact on the material property Some models relying on different backgrounds have been developed, such as mean-field and full-field models. Mean-field models give information about final mean grain size, whereas full-field models focus on the grain size distribution and the evolution of mean grain size as well. In this article, we examined the differences in those models for pure grain growth simulation by carrying out two simulations using DIGIMU 3.0 software that works with the full-field approach. For two simulations, different grain size distributions were generated by keeping the mean grain size constant while changing the standard deviation. If we use the mean-field models instead, we could already expect that the final mean grain sizes will be the same since we provide equal initial mean grain sizes. However, we also provide the grain size distribution, which makes two microstructures differ from each other. Therefore, we could track the grain size distribution during the grain growth thanks to the full-field approach. The main idea is to check the reliability of the mean-field model by investigating it from a wider window. At the end of the simulations, we found the final mean grain sizes are slightly different from each other, which proves the mean-field models are reliable are applicable to yield similar results with a full-field model while providing lower time consumption. However, simulations are following different paths during grain growth. However, it should be noted that mean-field models won't be applicable to multi-model grain size distributions and abnormal grain growth predictions. Therefore, the model should be selected according to the goal of the simulation.

\section{ACKNOWLEDGEMENT}

The author wish to thank Anıl Hatiboğlu (Middle East Technical University) for his enormous support on processing the simulation data.

\section{References}

1. Raabe, D. Recovery and Recrystallization: Phenomena, Physics, Models, Simulation, Editor(s): David E. Laughlin, Kazuhiro Hono, Physical Metallurgy (Fifth Edition), Elsevier, 2014, Pages 2291-2397, ISBN 9780444537706, https:// doi.org/10.1016/B978-0-444-53770-6.00023-X.

2. Atkinson, H. V. (1988). Overview no. 65: Theories of normal grain growth in pure single phase systems. Acta Metallurgica, 36(3), 469-491. 3. Strunk JrW, White EB. The Elements of Style, fourth ed. Longman, New York, 2000.

3. Goussery, V., Bienvenu, Y., Forest, S., Gourgues, A., Colin, C., \& Bartout, J. (2004). Grain size effects on the mechanical behavior of open-cell nickel foams. Advanced Engineering Materials, 6(6), 432-439. https://doi.org/10.1002/ adem.200405153

4. Kahn, M. (1971). Influence of grain growth on dielectric properties of NB-doped BaTiO3. Journal of the American Ceramic Society, 54(9), 455-457. https://doi. org/10.1111/j.1151-2916.1971.tb12384.x

5. M.M. Syazwan, A.N. Hapishah, R.S. Azis, Z. Abbas, M.N. Hamidon, Grain growth effects on magnetic properties of Ni0.6Zn0.4Fe204 material prepared using mechanically alloyed nanoparticles, Results in Physics, Volume 9, 2018, Pages 842-850, ISSN 2211-3797, https://doi. org/10.1016/j.rinp.2018.03.054.

6. Miyoshi, E., Takaki, T., Ohno, M., Shibuta, Y., Sakane, S. Shimokawabe, T., \& Aoki, T. (2017). Ultra-large-scale phase-field simulation study of ideal grain growth. NPJ Computational Materials, 3(1), 1-6

7. Kim, B. N., Hiraga, K., \& Morita, K. (2003). Kinetics of normal grain growth depending on the size distribution of small grains. Materials transactions, 44(11), 2239-2244.

8. Maire, L., Scholtes, B., Moussa, C. et al. Improvement of 3D mean field models for capillarity-driven grain growth based on full field simulations. J Mater Sci 51, 10970-10981 (2016). https://doi.org/10.1007/s10853-016-0309-6

9. Scholtes, B., Settefrati, A., Bozzolo, N., Perchat, E., Chenot, J. L., \& Bernacki, M. (2016, July). Large scale FE simulations of recrystallization and grain growth thanks to a level set approach, illustrations in context of industrial forming processes. NUMIFORM 2016: The 12th International Conference on Numerical Methods in Industrial Forming Processes, The Minerals, Metals \& Materials Society, Troyes, France. (hal-01421648)

10. Scholtes, B., Shakoor, M., Settefrati, A., Bouchard, P., Bozzolo, N., \& Bernacki, M. (2015). New finite element developments for the full field modeling of microstructural evolutions using the level-set method. Computational Materials Science, 109, 388-398. https://doi. org/10.1016/j.commatsci.2015.07.042

11. Furstoss, J., Bernacki, M., Petit, C., Fausty, J., Pino-Mu noz, D., \& Ganino, C. (2020). Full field and mean field modeling of grain growth in a multiphase material under dry conditions: application to peridotites. Journal of Geophysical Research: Solid Earth, e53942.

12. Mahadevan, S., Nalawade, S., Singh, J. B., Verma, A., Paul, B., \& Ramaswamy, K. (2012). Evolution of $\delta$ Phase Microstructure in Alloy 718 . Superalloy 718 and Derivatives, 737-750.

13. SAE International in United States, September 1965 [Aerospace Material Specification] AMS5663. 